\title{
On Some Myths about Sequenced Common-Value Auctions
}

\author{
Maria Angeles de Frutos \\ Departamento de Economia, Universidad Carlos III de Madrid, Madrid, Spain \\ and \\ Robert W. Rosenthal \\ Department of Economics, Boston University, Boston, Massachusetts, 02215
}

\begin{abstract}
Equilibria are constructed for classes of game models of sequenced second-price auctions having identical common-valued objects. In some of these the equilibrium price falls on average, and in others the seller loses on average by committing to announce publicly something that he knows. Both of these possibilities are surprises. Journal of Economic Literature Classification Number: D44.
\end{abstract}

\section{INTRODUCTION}

Most of auction theory is based on symmetric-game models in which bidders observe private signals from an ordered set and, in (pure-strategy) equilibrium, bid the same strictly monotonic function of their signals. (See, for instance, the survey of Milgrom (1985).) Some symmetric auction games cannot possibly have such equilibria, however. Consider the following class:

Two objects, known to be identical, are to be sold by a single seller at a pair of sealed-bid, second-price auctions with no reserve prices and no entry fees. The auctions occur one after the other, quickly enough that no participants discount anything, and the price paid in the first auction is announced publicly before the bidding in the second auction. There are at least three bidders, and each bidder desires only one of the objects; so the winner of the first auction does not participate in the second. Finally, each bidder's signal is a random variable affiliated with the identical true common values of the objects and, conditional on this true value, the signals are independent and have identical atomless distributions. 
No game in this class can have a symmetric equilibrium at which in the first auction each bidder bids the same strictly increasing function of her signal. To see the reason for this, note first that if there were such an equilibrium the bidder whose signal turns out to be second highest is at a major disadvantage in the second auction, since all other bidders can infer her signal (by inverting the bid function), whereas she knows only that their signals are lower and that one signal was higher. Consider, then, the position of a bidder in the first auction whose signal is very near, but does not quite equal, the lowest possible signal. Conditional on the (unlikely but germane) event that this signal is the first or second highest among all bidders, it is with very high probability the second highest. Consequently, this bidder has much more to gain than lose by deviating down to the lowest bid in the range of the equilibrium bid function. There can therefore be no symmetric equilibrium of this type. [In Bikhchandani's (1988) analysis of repeated second-price auctions, a reputation-building bidder bids aggressively to reveal his signal, as it will intensify the "winner's curse" for the other bidders and serve to deter competition subsequently. (Similar effects are found in Avery's (1994) jump-bidding equilibria of English auctions.) Here, by contrast, the effect is to bid cautiously to conceal information that could be used by opponents in subsequent auctions.]

We do not know what equilibria may look like for all of the games in the above class, but we present below some equilibria for examples from a related class in which signals can take on only two values, and, not surprisingly given the above, these equilibria involve pooling: bidders with different signals sometimes make the same bid in the first auction. (Some equilibrium bids are also randomized, but this comes more from the discrete nature of the signal space than from the rest of the auction structure.) Perhaps more importantly, however, some of the qualitative features of these equilibria run counter to what might be expected from the literature based on symmetric monotonic equilibria. (Much of the folk wisdom appears to come from Milgrom and Weber (1982b), a collection of widely circulated preliminary results and conjectures.) In particular, it is not always true that

1. The more information the seller can credibly reveal, the better for the seller (weakly).

2. The price in the second auction is on average higher (weakly) than the price in the first auction.

3. The more bidders there are, the better (weakly) for the seller. 
The rest of the paper is devoted to the examples. In Section 2, we construct a symmetric partial-pooling equilibrium for each member of a symmetric class of these games. We then construct symmetric equilibria for another class of games that is identical to the first class, except that the price in the first auction is not announced before the second. We show numerically that the seller can be made either better or worse off on average, depending on parameter values, by announcing the first-auction price. (Perry and Reny (1997) have recently provided another example in which the seller is unambiguously made worse off when he commits to reveal what he knows. Their set-up, however, is different from the one in this paper.) Thus the price in the first auction is a piece of information the seller may not wish to commit to revealing before the second auction. This runs counter to the intuition that by revealing information the seller ameliorates the "winner's curse." That intuition is evidently incomplete in cases like this one, at least, where the seller's information is partly endogenous.

In Section 3, we exhibit an equilibrium for an asymmetric game of a similar sort in which price falls in expectation not only conditional on some first-auction price realizations, but in a stronger ex ante expected sense as well. Although "afternoon effects" are commonly observed in practice (e.g., Ashenfelter, 1989), their possibility runs counter to two intuitive arguments about equilibria: (1) that there is increased competition (and hence higher prices on average) in the second auction after both one of the objects and one of the bidders have been removed, since the ratio of bidders to objects is now higher; and (2) that if expected prices in the two auctions were not equal, ex ante, a bidder could do better by bidding less aggressively in one of the auctions and more aggressively in the other. The example in Section 3 also has the feature that fewer bidders can make the seller better off, although this feature can be observed in simpler singleobject auctions as well.

Section 4 contains additional discussion, and some details of the arguments from Sections 2 and 3 are left to the Appendix.

Aside from exposing the falsity of some myths through special examples, there may be an additional benefit in presenting the equilibrium constructions of this paper. The question of how best to auction multiple objects has been of great interest lately, and the answer has been elusive, primarily because the construction of equilibria under alternative scenarios has been limited to some very simple examples. (The recent spectrum auctions of the Federal Communications Commission in the U.S. have sparked interest in the subject most recently. McMillan (1994) discusses the design issues faced by the F.C.C.) The constructions of this paper may aid in the search for a general construction that can be used for sequential designs. 


\section{SYMMETRIC EXAMPLES}

There are three players. At the beginning of the game, each player secretly observes the signal 1 with probability $q$ and the signal 0 with probability $(1-q)$. The players' signals are independent of each other. The value of either of the two objects to be auctioned to each of the players is the sum of the signals. No player wants more than one object, however, and the winner of the first auction is excluded from the second. The rules are sealed-bid, second-price in both auctions, with the usual unbiased tie-breaking rule. (Sealed-bid, second-price auctions are rare. The literature's interest in them stems from the similarity of some of their theoretical properties to those of the widely used, but more complicated, English auctions. That similarity is less compelling here: in a pair of sequenced English auctions, for instance, the seller would be unable to keep secret the winning bid of the first auction.)

\subsection{Price-Announced Case}

When the second highest bid in the first auction is announced before the second auction, a pure strategy in the game is for each of the two possible signals (types): a bid in the first auction together with a function mapping announced prices to bids for the second game.

For $q \in(0,1)$, let

$$
p(q) \equiv \frac{2 q-2+\sqrt{(1-q)(4-q)}}{q} .
$$

Note that $p(q)$, which is the equilibrium pooling probability for the 1-types in the first auction, declines from 0.75 to 0 as $q$ goes from 0 to 1 . The following behavioral strategy forms a symmetric equilibrium.

0-Type

First auction: Bid

$$
b(q) \equiv \frac{2 q p(q)}{q p(q)+1-q} .
$$

Second auction: Bid

$$
\begin{cases}e(q) \equiv \frac{3 q-2 q p(q)}{2 q-2 q p(q)+1} & \text { if the 1st-auction price was }=b(q) \\ 2 & \text { if the 1st-auction price was }>b(q) .\end{cases}
$$

1-Type 
First auction: With probability $p(q)$ bid $b(q)$, and with probability $1-p(q)$, randomize according to the cumulative distribution function (c.d.f.)

$$
\begin{aligned}
Z_{q}(x) \equiv \frac{(x-2)(1-q)-(3-x) q p(q)}{(3-x) q(1-p(q))} \\
\text { on }\left[\frac{2-2 q+3 q p(q)}{1-q+q p(q)}, 2+q\right] .
\end{aligned}
$$

(It is easy to check that $Z_{q}$ is a genuine c.d.f. for each $q$ and that the support of $Z_{q}$ always lies above 2, while $b(q)$ is always below 1; so a 1-type who does not pool with the 0-types always outbids both the 0-types and the pooled 1-types in the first auction.)

Second auction: If the first-auction price was greater than own bid, bid 3; if the first-auction price was equal to own bid, which was greater than $b(q)$, bid 3; if the first-auction price was equal to own bid, which was less than or equal to $b(q)$, bid

$$
c(q) \equiv \frac{7 q+2-6 q p(q)}{2 q+1-2 q p(q)} .
$$

(Note that $e(q)<1$ and $2<c(q)<3$, so a 1-type who pooled in the first auction will always outbid a 0 -type in the second auction.)

At this equilibrium, the 0-types break even on average in both auctions, competing away the profits in Bertrand fashion. The 1-types who do not pool are the only possible winners in the first auction whenever they are present. Higher bids in the support of $Z_{q}$ increase a 1-type's chance of winning in the first auction, but this is offset by the higher expected price involved. The pooling bid $b(q)$ equates the expected profit from winning the first auction (and paying $b(q)$ ) with losing (with its chances of winning the second auction and paying only $e(q)<b(q)$ ). The pooled 1-types receive lower expected payoffs than the nonpooled 1-types in the first auction, but compensate with higher expected payoffs in the second auction. The argument that this strategy forms a symmetric equilibrium is found in the Appendix. We conjecture that it is the only symmetric one. Expected prices in the various possible events can be found in Table I (where dependence on $q$ is suppressed and where $E Z_{i: j}$ denotes the expectation of the $i$ th-order statistic from a random sample of size $j$ distributed according to the c.d.f. $Z$ ).

Since the order statistics from $Z$ always lie between 2 and 3, it is obvious from this table that conditional on the first-auction price, the expected 
TABLE I

\begin{tabular}{|c|c|c|c|c|}
\hline Prob. & Prob. & Auction 1 price & Prob. & Auction 2 price \\
\hline$(1-q)^{3}$ & & $b$ & & $e$ \\
\hline \multirow[t]{2}{*}{$3(1-q)^{2} q$} & & $b$ & & $e$ \\
\hline & $\left((1-p)^{2}\right.$ & $E Z_{2: 2}$ & & 2 \\
\hline \multirow[t]{3}{*}{$3(1-q) q^{2}$} & $2 p(1-p)$ & $b$ & & $e$ \\
\hline & $1 p^{2}$ & $b^{b}$ & $\left\{\begin{array}{l}1 / 3 \\
2 / 3\end{array}\right.$ & $c$ \\
\hline & $\left((1-p)^{3}\right.$ & $E Z_{2: 3}$ & & 3 \\
\hline \multirow[t]{3}{*}{$q^{3}$} & $\left\{3(1-p)^{2} p\right.$ & $E Z_{2: 2}$ & & 3 \\
\hline & $3(1-p) p^{2}$ & $b$ & & $c$ \\
\hline & $p^{3}$ & $b$ & & $c$ \\
\hline
\end{tabular}

second-auction price can be either higher or lower than the first-auction price. Some algebra reveals that the ex ante expected prices are

$$
\begin{gathered}
E\left(p_{1}\right)=6 q^{3}-39 q^{2}+72 q-36+\left(6 q^{2}-24 q+18\right) \sqrt{4-5 q+q^{2}} \\
E\left(p_{2}\right)=-12 q^{3}+60 q^{2}-81 q+36 \\
-\left(12 q^{2}-30 q+18\right) \sqrt{4-5 q+q^{2}}
\end{gathered}
$$

These are graphed against $q$ in Fig. 1, from which we see that the price does rise in the ex ante sense.

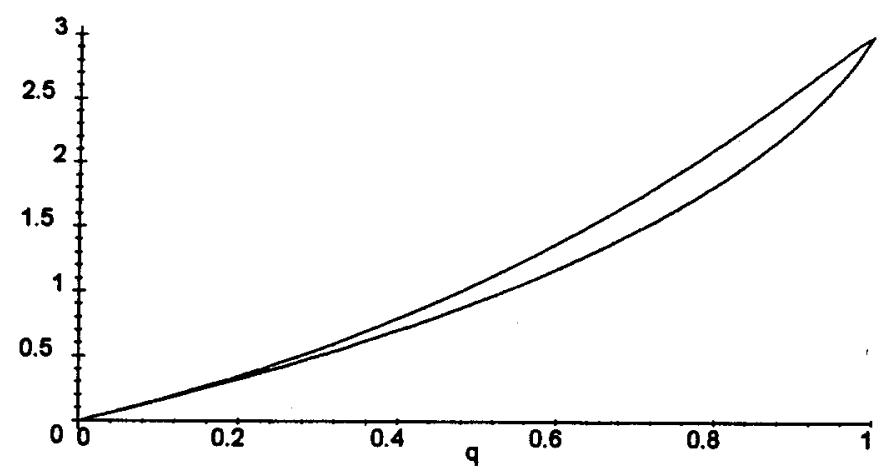

FIG. 1. $E\left(p_{1}\right) \leq E\left(p_{2}\right)$. 


\subsection{Price-Not-Announced Case}

When the second-highest bid in the first auction is not announced, a pure strategy is an ordered pair of bids-one for each auction. The following behavioral strategy forms a symmetric equilibrium.

0-Type

First auction: Randomize according to the c.d.f. $F_{q}$, which is the unique function satisfying

$$
\ln (1-(1-q) F(x))=-\frac{(1-q) x F(x)}{q} \quad \text { on }\left[q, \frac{-q \ln q}{1-q}\right] .
$$

(That $F_{q}$ is a genuine c.d.f. for each $q$ is verified in the Appendix.) lost), bid

Second auction: If the first-auction randomization produced $x$ (and

$$
g_{q}(x) \equiv \frac{q}{1-(1-q) F_{q}(x)} .
$$

(Note that $g_{q}$ is monotone increasing, that $g_{q}(q)=q$, and that $g_{q}(-q \ln q /(1-q))=1$.)

1-Type

First auction: Randomize according to the c.d.f.

$$
H_{q}(x) \equiv \frac{(1-q) x+q \ln q}{q(3-x)} \quad \text { on }\left[\frac{-q \ln q}{1-q}, 3 q-q \ln q\right] \text {. }
$$

(That $H_{q}$ is a genuine c.d.f. for each $q$ is straightforward to check.) Second auction: Bid 3.

In this equilibrium, there is no pooling: the 1-types always outbid the 0 -types. The 0-types again break even on average in the pair of auctions, but not in each auction individually: those who bid higher in the first auction do worse on average in the first auction when they win it, but they profit more on average in the second auction from the informational rent they gain when they lose in the first auction at a higher bid. (Randomization over linked pairs of bids is also found in the equilibria of Lang and Rosenthal (1991). The interpretation of the linking there is somewhat different, however.) For the 1-types, a loss in the first auction must have been to another 1-type, so a bid of 3 in the second auction is best against the strategy in question. The randomization in the first auction simply trades off higher win probabilities against higher prices, with proper accounting for the expected profits in the second auction after a loss in the 
first. Notice that the bottom of the support of $H$ is exactly the same as the top of the support of $F$.

The argument that this strategy forms a symmetric equilibrium is found in the Appendix. Again we think it is the unique symmetric one. (With a different tie-breaking rule, however, there is at least one more, at least when $q \leq 0.25$. See Section 4.) Expected prices in the various possible events can be found in Table II (where dependence on $q$ is again suppressed).

Again, it is easy to see that conditional on the first-auction price, the expected second-auction price can be either higher or lower. Some algebra (see the Appendix) reveals, however, that ex ante the expected prices in both auctions are exactly the same, namely $3 q\left(1+q^{2}\right) / 2$.

\subsection{Revenue Comparisons}

The expected seller's revenue is the sum of the ex ante expected prices. In the price-announced case, this is

$$
-6 q^{3}+21 q^{2}-9 q-\left(6 q^{2}-6 q\right) \sqrt{4-5 q+q^{2}} .
$$

In the price-not-announced case, it is simply $3 q\left(1+q^{2}\right)$. In Fig. 2 the difference in the expected seller's revenue between the first and second cases is graphed against $q$. Figure 2 reveals that the revenue ranking switches near 0.80 , so when $q$ is high, the seller does better by committing to not revealing the first-auction price.

\section{AN ASYMMETRIC EXAMPLE}

In this section, there are only two possible common values, 0 and 1 , for the identical objects. A set of two informed bidders knows the true value $V$. A set of two uninformed bidders knows only that both values are

TABLE II

\begin{tabular}{ccc}
\hline Prob. & Auction 1 price & Auction 2 price \\
\hline$(1-q)^{3}$ & $E F_{2: 3}$ & $E g F_{3: 3}$ \\
$3(1-q)^{2} q$ & $E F_{1: 2}$ & $E g F_{2: 2}$ \\
$3(1-q) q^{2}$ & $E H_{2: 2}$ & $E g F$ \\
$q^{3}$ & $E H_{2: 3}$ & 3 \\
\hline
\end{tabular}

$E g F_{i: j}$ denotes the expectation of $g$ of the $i$ th of $j$-order statistics from $F$ and $E g F$ denotes the expectation of $g$ of a single draw from $F$. 


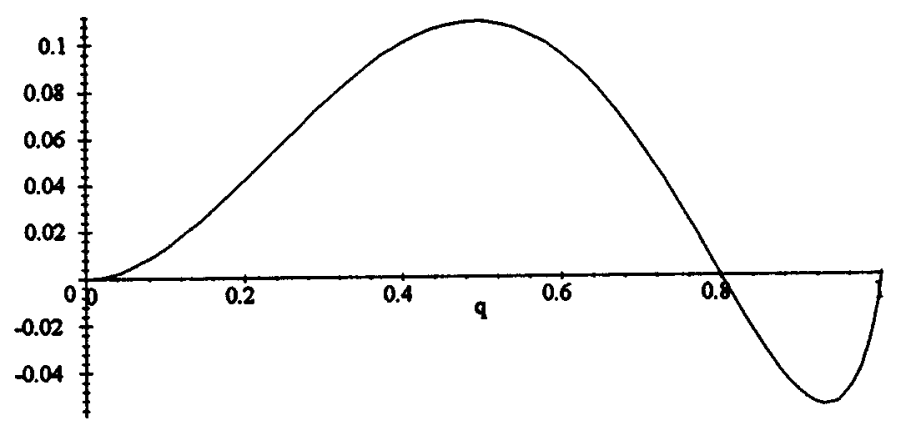

FIG. 2. Revenue difference.

equally likely. We assume first that the price in the first auction is not announced before the second auction.

We restrict attention to strategies in which, from familiar dominance considerations, in the second auction the informed bid $V$ and the uninformed bid 0 ; so only the first-auction bids need to be derived. (Note that there are non-type-symmetric equilibria in which one informed bidder bids 0 in the first auction while the other bids $V$, and the uninformed all bid 0 . Hence, since there is at most one nonzero bidder in each auction, the prices are constant at 0 in these non-type-symmetric equilibria.) At the unique equilibrium of interest, the c.d.f. for the bid of the 1-informed in the first auction is

$$
I(x)= \begin{cases}\frac{x}{\sqrt{4-7 x+3 x^{2}}} & \text { if } 0 \leq x<\frac{7-\sqrt{17}}{4} \\ 1 & \text { if } x \geq \frac{7-\sqrt{17}}{4}\end{cases}
$$

whereas the c.d.f. of the bid of the uninformed is

$$
U(x)=\left\{\begin{array}{ll}
\frac{(\sqrt{17}-3)(4-3 x)}{(3 \sqrt{17}-5)(1-x)} e^{(1 /(3-\sqrt{17}))+(1 /(4-4 x))} & \text { if } 0 \leq x<\frac{7-\sqrt{17}}{4} \\
1 & \text { if } x \geq \frac{7-\sqrt{17}}{4}
\end{array} .\right.
$$

(The proof of this is in the Appendix; the functions are plotted in Figs. 3 and 4.) 


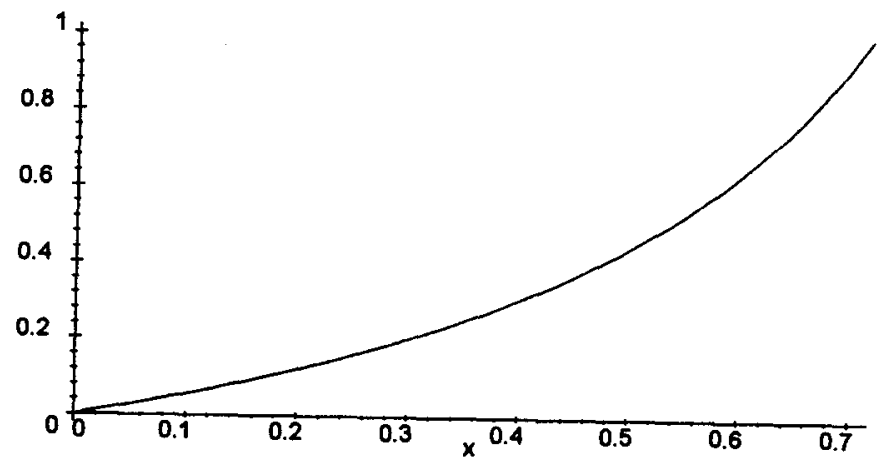

FIG. 3. $I(x)$.

The expected price in the first auction is

$$
\begin{aligned}
& \left.\int_{0}^{(7-} \sqrt{17}\right) / 4 x(I(x))^{2}(1-U(x)) U^{\prime}(x) d x \\
& \quad+2 \int_{0}^{(7-\sqrt{17}) / 4} x I(x)(1-I(x)) U(x) U^{\prime}(x) d x \\
& \quad+2 \int_{0}^{(7-\sqrt{17}) / 4} x I(x) U(x)(1-U(x)) I^{\prime}(x) d x \\
& \quad+\int_{0}^{(7-\sqrt{17}) / 4} x(U(x))^{2}(1-I(x)) I^{\prime}(x) d x \\
& \quad+\int_{0}^{(7-\sqrt{17}) / 4} x(1-U(x)) U^{\prime}(x) d x .
\end{aligned}
$$

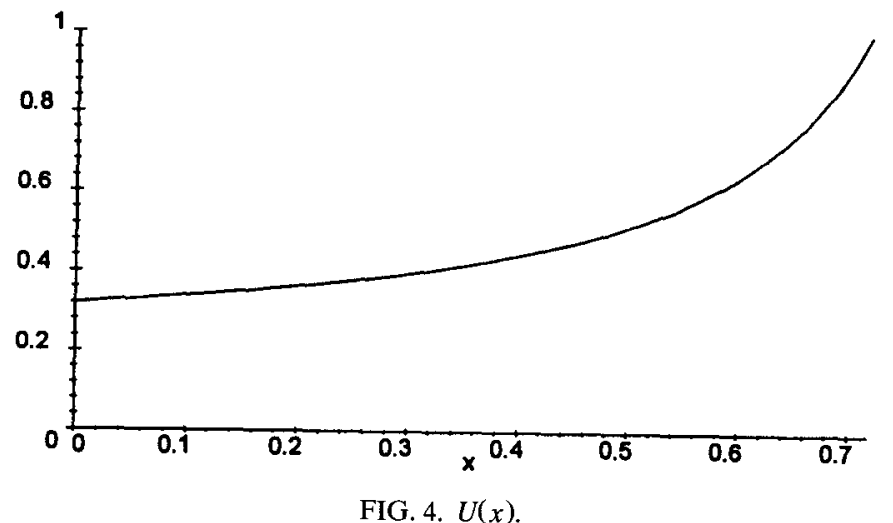


The first four terms above are for the case $V=1$; the fifth term is for $V=0$. The expected price in the second auction is just the probability that $V=1$ and an uninformed wins the first auction:

$$
\int_{0}^{(7-\sqrt{17}) / 4} I(x)\left(1-(U(x))^{2}\right) I^{\prime}(x) d x .
$$

Numerical evaluations produce 0.38 for the first auction and 0.24 for the second, so the ex ante expected price declines.

Next, notice that if the price in the first auction is announced before the second auction, the equilibrium described above remains an equilibrium in the sense that all players ignore the announcement and bid as above. An additional type-symmetric equilibrium is present in the price-announced game, however. In it, both informed bid $V$ in both auctions, whereas the uninformed bid 0 in the first auction and bid the announced first-auction price in the second. This equilibrium is better for the seller and possesses no "afternoon effect," of course.

Finally, notice that if there is only one uninformed bidder, there is an equilibrium for the price-not-announced game in which the uninformed bids 1 in both auctions, whereas the informed both bid $V$ in both auctions. This means the seller earns $2 V$. On average, therefore, the seller earns 1 in total, which is more than at the randomized equilibrium above. Of course, if there are at least three informed, the seller again earns $2 \mathrm{~V}$ at the obvious equilibrium.

\section{CONCLUDING REMARKS}

1. That there should be no upward or downward trend in prices over time on average in sequenced auctions seems to be a well-understood property of certain environments. For instance, in an independentprivate-values setting, Weber (1983) studies the sequential sale of $k$ objects to risk-neutral bidders who each desire only one object. He shows that the equilibrium price sequence is a martingale. The result is established with an arbitrage argument: if the sequence of prices were, for instance, downward drifting, then, in equilibrium, bidders with high values would bid low in the first auction, preferring to bid more aggressively later. But this behavior produces higher prices later, contradicting the assumed downward drifting sequence. (The risk-neutrality assumption is important for this result: if bidders were risk averse, then the sequence of prices could well be downward drifting, as the arbitrage argument would be complicated when a bidder incorporates in his current bid the risk associated with future prices (see McAfee and Vincent, 1993). Other variations 
of Weber's model also have declining prices, e.g., Bernhardt and Scoones (1994) and Gale and Hausch (1992).)

2. A similar arbitrage argument helps explain our finding that ex ante expected prices are equal for the two auctions in the price-notannounced case of Section 2.2: Although our model has common values, when the price is not announced, no information about other bids is revealed from a loss in the first auction beyond what an individual could deduce for herself a priori. Hence, if the ex ante expected price path were not constant, bidders would have incentives to bid more aggressively in the auction having the lower price and higher in the other auction.

3. Information revelation considerations are generally more important for sequential sales of common-valued objects than for the privatevalues model. To the extent that the equilibrium is separating under common values, bids reveal information that can be used subsequently. Even if the price is not announced, a bidder who has lost in the first auction has more information at the beginning of the second auction about the common value than she had originally. (If there were a monotone, symmetric equilibrium, for instance, she would know that there is a bidder with a higher estimate than hers.) In the private-values case, such information is worthless; but in the common-values case, it must be taken into account in computing the optimal bid. Thus, in Weber (1983), the symmetric equilibrium strategies for a sequential second-price auction are actually the same whether the price is announced or not. Of course, this is not generally the case for the common-values case. (See, however, Section 3.)

4. In a single-object, common-values setting, Milgrom and Weber (1982a) show that the seller can raise expected revenue by committing to a policy of publicly releasing whatever information he learns. The released information adds an additional link between a bidders's information and the selling price, and thereby reduces the "winner's curse." Thus players are willing to bid more aggressively on average, which is advantageous to the seller. Matters are not so simple in the case of sequential sale, however. If a player knows that his bid may reveal information, then he has an incentive to dissemble.

5. The asymmetric model in Section 3 illustrates a sense in which the seller is made worse off by the presence of additional bidders. An even simpler instance of it can be seen in a single-object, common-value model (again sealed-bid, second-price auction rules) in which the object is worth 0 or 1 with equal prior probabilities. (The phenomenon is familiar from Rosenthal (1980). We are grateful to Ruqu Wang for this example.) If it is common knowledge that there are two bidders and they are both uninformed, then at the unique equilibrium they both bid $1 / 2$. If a third bidder is added who knows the true value, at the unique type-symmetric equilib- 
rium he bids the true value and the uninformed bid 0 . Thus the presence of the informed bidder destroys the competitive pressure that was present in the two-bidder case.

6. For the price-not-announced model of Section 2.2, with a different tie-breaking rule there is at least one additional symmetric equilibrium when $q \leq 0.25$. The rule is: If a tie involves more 1-types than 0-types, then the 0 -types lose and the tied 1-types win with equal probabilities. If a tie involves at least as many 0 -types as 1-types, then the 1-types lose and the tied 0 -types win with equal probabilities. (This rule is not usable unless the auctioneer can observe the types, but the example may be interesting anyway, in light of the existence result in Simon and Zame (1990).) The type-symmetric equilibrium strategy is: In the first auction, all bidders bid $3 q /(2 q+1)$; in the second auction, all 0 -types bid 0 and all 1-types bid 3 . We leave the verification to the reader.

7. This paper leaves open several interesting questions. These include:

i. When bidders' signals can take on values in a continuum, since the standard monotone-bid equilibrium constructions do not work in our sequential setting, what does work?

ii. Under what conditions are simultaneous designs superior for the seller (cf. Rosenthal and Wang, 1996)?

iii. What information about early auctions is it optimal for the seller to reveal later?

\section{APPENDIX}

\section{Equilibrium from Section 2.1}

Again, we suppress dependence on $q$ for convenience. For a 0-type in the second auction, if the price was $>b$ in the first auction, the other two bidders must be 1-types, and so the object is worth 2 . By the usual argument in second-price single-object auctions, therefore, the bidder must bid 2. If the price was $b$ in the first auction, a bid of $e$ earns in expectation

$$
(1-e) 2 q(1-q)\left(1-\frac{2 p}{3}\right) \frac{1}{2}+(-e)(1-q)^{2} \frac{2}{3} \frac{1}{2},
$$

which is 0 . A bid below $e$ always loses, so earns 0 ; a bid in $(e, c)$ changes the tie-breaking factors of $1 / 2$ in the above to 1 and so again produces zero expected payoff. A bid of $c$ or more is weakly dominated by a bid of 2 , since the objects can be worth no more than 2 , and so cannot result in 
positive expected payoff. So there are no profitable deviations by a 0 -type in the second auction, where 0 payoffs are expected.

For a 0-type in the first auction, a bid of $b$ earns in expectation

$$
(2-b) q^{2} p^{2} \frac{1}{3}+(1-b) 2 q p(1-q) \frac{1}{3}+(-b)(1-q)^{2} \frac{1}{3}=0 .
$$

A bid below $b$ always loses and leads to zero expected payoffs in the second auction. A bid above 2 is again dominated by a bid of 2 . From the last equation, a bid between $b$ and 2 breaks even when it wins, and produces no information that can be profitably used in the second auction when it loses, and so there are no profitable deviations by a 0-type.

For a 1-type in the second auction, if the first-auction price exceeded the bidder's own bid, the other two bidders must be 1-types, and so the object is worth 3 . The bidder must bid 3 for the usual reasons. If in the first auction the player's bid set the price and was above $b$, by bidding 3 he bids the true value when that value is 3 , and ensures winning and paying 2 when that value is 2 , so the usual argument applies again. For the event when the price and his bid were both $b$ in the first auction, the expected payoff from bidding $c$ in the second auction is

$$
\begin{gathered}
(3-c) q^{2}\left(p^{2} \frac{2}{3} \frac{1}{2}+2 p(1-p) \frac{1}{2}\right)+(2-c) 2 q(1-q) p \frac{1}{3} \frac{1}{2} \\
+(2-e) 2 q(1-q)\left(1-p+\frac{p}{3}\right)+(1-e)(1-q)^{2} \frac{2}{3}
\end{gathered}
$$

The value chosen for $c$ makes the first two terms above sum to 0 ; so deviations into $(e, c)$, which eliminate these two terms, make no payoff difference, and deviations into $(c, 3)$, which change all of the (1/2)-tie-break probabilities in these two terms to 1, also make no payoff difference. Bids less than or equal to $e$ and equal to 3 obviously do no better.

For a 1-type in the first auction, a bid of $b$ produces the expected payoffs:

$$
\begin{array}{r}
(3-b) q^{2} p^{2} \frac{1}{3}+(2-b) 2 q(1-q) \frac{p}{3}+(1-b)(1-q)^{2} \frac{1}{3} \\
+(2-e) 2 q(1-q)\left(1-p+\frac{p}{3}\right)+(1-e)(1-q)^{2} \frac{2}{3}
\end{array}
$$

Substituting for $b$ and $e$ and simplifying, this becomes

$$
\frac{1}{3} q^{2} p^{2}-\frac{2}{3} p q(1-q)+1-q^{2} .
$$


For a 1-type in the first auction, a bid of $x>b$ produces the expected payoff:

$$
\begin{aligned}
& q^{2} \int_{b}^{x}(3-y)\left[(1-p)^{2} 2 Z(y) Z^{\prime}(y)+2 p(1-p) Z^{\prime}(y)\right] d y \\
& \quad+2 q(1-q)(1-p) \int_{b}^{x}(2-y) Z^{\prime}(y) d y \\
& \quad+(3-b) q^{2} p^{2}+(2-b) 2 q(1-q) p+(1-b)(1-q)^{2} .
\end{aligned}
$$

For bids in the support of $Z$ to produce equal payoffs, therefore,

$$
\begin{gathered}
(3-x) q^{2}\left[(1-p)^{2} 2 Z(x) Z^{\prime}(x)+2 p(1-p) Z^{\prime}(x)\right] \\
+(2-x) 2 q(1-q)(1-p) Z^{\prime}(x)=0,
\end{gathered}
$$

which is satisfied uniquely by the $Z$ defined earlier. In this case, the expected payoff from bidding in this support is given by its value at the lower limit of the support, namely,

$$
(3-b) q^{2} p^{2}+(2-b) 2 q(1-q) p+(1-b)(1-q)^{2} .
$$

Substituting for $b$, this becomes

$$
q^{2} p^{2}+2 q p-2 q^{2} p+(1-q)^{2} .
$$

Equating (1) and (2) generates the quadratic equation whose (relevant) solution is $p$, defined earlier.

Since $Z$ is atomless, deviations above its support do no better than bidding the upper limit of that support, and deviations below its support are no better than the lower limit. Deviations below $b$ are obviously worse than bidding $b$.

\section{Equilibrium from Section 2.2}

To compute $g(x)$, pick $r$ to maximize expected second-auction profit for a 0-type when the first-auction companion bid is $x$ from the support of $F$ and when the other bidders bid according to the hypothesized strategy:

$$
\begin{aligned}
(1-q)^{2} \int_{z=q}^{g^{-1}(r)}(-g(z)) 2(1-F(x)) F^{\prime}(z) d z \\
+2 q(1-q) \int_{z=q}^{g^{-1}(r)}(1-g(z)) F^{\prime}(z) d z .
\end{aligned}
$$


Differentiating with respect to $r$, equating the result to zero, and setting $g(x)=r$ produces

$$
(1-q)(-g(x))(1-F(x))+q(1-g(x))=0,
$$

and hence the function $g$ in Section 2.2. To see that this $g(x)$ is the best companion in the second auction to the bid $x$ from the support of $F$ in the first auction, consider first an alternative $r \in(g(x), 1]$. The difference between the payoff to the pair of bids $x$ and $r$ and the pair $x$ and $g(x)$ is the expression in (3) with the lower limits of the integrals replaced by $x$. Substituting the expression for $g(\cdot)$ from Section 2.2, this is clearly negative. A similar argument takes care of a companion bid $r \in[q, x)$. A companion bid $\in(1,3)$ is no better than 1 , a companion below $q$ is no better than $q$, and a companion of 3 or more is obviously hopeless.

Now, assuming that the companion bid is $g(x)$, the break-even condition for the 0-types in the first auction is

$$
\begin{aligned}
& (1-q)^{2} \int_{z=q}^{x}(-z) 2 F(z) F^{\prime}(z) d z \\
& \quad+(1-q)^{2} 2(1-F(x)) \int_{z=q}^{x}(-g(z)) F^{\prime}(z) d z \\
& \quad+2 q(1-q) \int_{z=q}^{x}(1-g(z)) F^{\prime}(z) d z=0 .
\end{aligned}
$$

This clearly holds at $q$. It will be satisfied on $(q,(-q \ln q) /(1-q)]$ if its derivative is zero there. This condition on the derivative (simplified) is

$$
\begin{aligned}
& (1-q)(-x) F(x) F^{\prime}(x)+(1-q)(-g(x))(1-F(x)) F^{\prime}(x) \\
& \quad-F^{\prime}(x)(1-q) \int_{z=q}^{x}\left(-g(z) F^{\prime}(z)\right) d z+q(1-g(x)) F^{\prime}(x)=0 .
\end{aligned}
$$

Substituting for $g(\cdot)$ results in the equation

$$
-x F(x)+\int_{z=q}^{x} \frac{q F^{\prime}(z)}{1-(1-q) F(z)} d z=0 .
$$

Or

$$
-x F(x)-\frac{q}{1-q} \ln (1-(1-q) F(x))=0 .
$$

For $x<q$, the only solution to this functional equation is $F(x)=0$. But above $q$, another, positive solution exists, and the locus of these positive 
solutions is increasing and continuous in $x$. (To see this, substitute $z=$ $1-(1-q) F$ and write the equation for the inverse function as $x=$ $-q \ln z /(1-z)$. It is not hard to check that this $x$ is continuous and monotone decreasing in $z$ on $[q, 1]$.) The expression for the upper limit of the support of $F$ is obtained by setting $F(x)=1$ and solving for $x$.

A deviation by a 0 -type in the first auction below $q$ with any secondauction companion is no better than $q$ with that same companion. To see if the 0-types would gain by deviating into the support of $H$, we calculate the derivative of a 0 -type's payoffs in this region (when the companion bid is one in the second auction, which has to be the best companion for such a deviation), and it is negative. This means that any such deviation (and hence any above the support of $H$, too) is no better than the deviation $-q \ln q /(1-q)$, which is itself unprofitable.

That the 1-types do best by bidding 3 in the second auction is obvious. To determine the 1-types' first-auction randomization with this companion, consider overall expected payoffs against equilibrium play by the others:

$$
\begin{aligned}
q^{2} \int_{z=}^{x} & (-q \ln q) /(1-q) \\
& +2 q(1-q) \int_{z=(-q \ln q) /(1-q)}^{x}(2-z) H^{\prime}(z) d z \\
& +(1-q)^{2} \int_{z=q}^{(-q \ln q) /(1-q)}(1-z) 2 F(z) F^{\prime}(z) d z \\
& +2 q(1-q)(1-H(x)) \int_{z=q}^{(-q \ln q) /(1-q)}(2-g(z)) F^{\prime}(z) d z .
\end{aligned}
$$

Next check that with the $g$ and $H$ specified in Section 2.2, overall expected payoffs are constant on the support of $H$. A deviation above the support does not gain relative to the top of the support, and a deviation to $x$ in the support of $F$ (with best companion 3 in the second auction) produces

$$
\begin{aligned}
2 q(1 & -q) \int_{z=q}^{(-q \ln q) /(1-q)}(2-g(z)) F^{\prime}(z) d z \\
& +(1-q)^{2} \int_{z=q}^{x}(1-z) 2 F(z) F^{\prime}(z) d z \\
& +(1-q)^{2}(1-F(x)) \int_{z=q}^{x} 2(1-g(z)) F^{\prime}(z) d z \\
& +(1-q)^{2} \int_{r=v}^{(-q \ln q) /(1-q)} 2(1-g(z))(1-F(z)) F^{\prime}(z) d z .
\end{aligned}
$$


Using the break-even conditions for the 0-types in the two auctions, the last three terms of (7) are seen to equal

$$
\begin{aligned}
& (1-q)^{2}\left(F^{2}(x)+2(1-F(x)) F(x)\right) \\
& -2 q(1-q) \int_{z=q}^{x}(1-g(z)) F^{\prime}(z) d z \\
& +(1-q)^{2}(1-F(x))^{2}-2 q(1-q) \\
& \quad \times \int_{z=x}^{(-q \ln q) /(1-q)}(1-g(z)) F^{\prime}(z) d z,
\end{aligned}
$$

which, after some algebra, just equals the third term in (6). Since the bidder is indifferent over the support of $H$, his expected payoff from not deviating can be calculated by evaluating (6) at $-q \ln q /(1-q)$. The first two terms of this expression vanish, and the fourth term becomes equal to the first term in (7). Altogether then, we have shown that a deviation into the support of $F$ (with best companion bid in the second auction) does no better (and no worse) than a bid in the support of $H$ (with companion 3 ). A deviation below this support is obviously no better than a bid at its lower limit.

\section{Expected prices at the equilibrium from Section 2.2}

First, $E g F=-q \ln q /(1-q)$; this follows from evaluating (5) at $x=$ $-q \ln q /(1-q)$. To show that

$$
E F_{1: 2}=2 q\left(\frac{1-q+q \ln q}{(-1+q)^{2}}\right),
$$

use the previous result and the break-even condition for the 0-types in the first auction at $x=-q \ln q /(1-q)$. Similarly, integrating (4) leads to $E g F_{2: 2}=E F_{1: 2}$.

Multiplying both sides of (4) by $(1-F(x)) F^{\prime}(x)$, substituting this into the definition of $E g F_{3: 3}$, and simplifying results in

$$
E g F_{3: 3}=1.5 q\left(\frac{-1+4 q-3 q^{2}+2 q^{2} \ln q}{(q-1)^{3}}\right) .
$$

To compute $E F_{2: 3}$, first note that

$$
E F_{2: 3}=3 E F_{1: 2}-6 \int_{z=q}^{(-q \ln q) /(1-q)} z F^{2}(z) F^{\prime}(z) d z .
$$


Substituting for $z F(z)$ by using (5), we get

$$
E F_{2: 3}=3 E F_{1: 2}-6 \int_{z=q}^{(-q \ln q) /(1-q)} F(z)\left(\int_{y=q}^{z} g(y) F^{\prime}(y) d y\right) F^{\prime}(z) d z .
$$

Integration by parts results in $E F_{2: 3}=E g F_{3: 3}$.

Finally, routine calculations show that

$$
3(1-q) q^{2} E H_{2: 2}+q^{3} E H_{2: 3}=3(1-q) q^{2}(E g F)+q^{3} 3 .
$$

Thus,

$$
E\left(p_{1}\right)=E\left(p_{2}\right)=1.5\left(q^{3}+q\right)
$$

\section{Equilibrium from Section 3}

We hypothesize an equilibrium in which the 0 -informed bid 0 in the first auction, the 1-informed bid according to the c.d.f. $I$, and the uninformed bid according to the c.d.f. $U$, the supports of both $I$ and $U$ being subsets of $[0,1]$. Assuming differentiability, except possibly at 0 , the expected payoff to a 1 -informed bidding $z \in(0,1]$ is

$$
\begin{aligned}
& I(z) U^{2}(z)\left[1-\frac{\int_{x=0}^{z} x I^{\prime}(x) U^{2}(x) d x+2 \int_{x=0}^{z} x I^{\prime}(x) U(x) I(x) d x}{I(z) U^{2}(z)}\right] \\
& \quad+\int_{x=z}^{1} I^{\prime}(x) U^{2}(x) d x .
\end{aligned}
$$

For indifference to hold on the interior of the support of $I$, the derivative of this expression with respect to $z$ must be 0 . Simplified, that condition becomes

$$
I^{\prime}(z)=\frac{2(1-z) I(z) U^{\prime}(z)}{z U(z)}
$$

Similarly, the expected payoff to an uninformed bidding $z \in(0,1]$ is

$$
\begin{aligned}
& \frac{1}{2} U(z)\left[\frac{-\int_{x=0}^{z} x U^{\prime}(x) d x}{U(z)}\right]+\frac{1}{2} I^{2}(z) U(z) \\
& \quad \times\left[1-\frac{2 \int_{x=0}^{z} x I^{\prime}(x) I(x) U(x) d x+\int_{x=0}^{z} x U^{\prime}(x) I^{2}(x) d x}{I^{2}(z) U(z)}\right] .
\end{aligned}
$$


Differentiating as above and simplifying results in

$$
U^{\prime}(z)=\frac{2 I(z) I^{\prime}(z) U(z)(1-z)}{z-I^{2}(z)+z I^{2}(z)} .
$$

Substituting the expression for $I^{\prime}$ into that for $U^{\prime}$ yields

$$
I^{2}(z)=\frac{z^{2}}{4-7 z+3 z^{2}} .
$$

Notice $I^{2}(0)=0$, and $I^{2}$ increases on $[0,6 / 7]$. To find the upper limit for the support, we set $z^{2}=4-7 z+3 z^{2}$, which produces $z=$ $(7 \pm \sqrt{17}) / 4$. Taking the upper limit of the support of $I$ to be

$$
\frac{7-\sqrt{17}}{4} \cong 0.72<6 / 7
$$

therefore, results in a c.d.f. Substituting the expression for $I$ back into the expression for $U^{\prime}$ generates the differential equation

$$
U^{\prime}(z)=\frac{(8-7 z) U(z)}{4(1-z)^{2}(4-3 z)} .
$$

Taking the initial condition to be $U((7-\sqrt{17}) / 4)=1$, standard arguments show that it has a unique solution on $[0,(7-\sqrt{17}) / 4]$ which is

$$
U(z)=\frac{(\sqrt{17}-3)(4-3 z)}{(3 \sqrt{17}-5)(1-z)} e^{(1 /(3-\sqrt{17}))+(1 /(4-4 x))},
$$

an increasing function with $U(0)>0$, and therefore a c.d.f. By construction, the 1-informed and the uninformed are indifferent over the supports of their mixtures. Neither type can gain by bidding either above $(7-\sqrt{17}) / 4$ or below 0 . Similarly, the 0 -informed cannot gain by making nonzero bids, so $I$ and $U$ generate an equilibrium.

That there can be no other type-symmetric equilibria in the game is easy to verify: Neither $I$ nor $U$ can have mass points other than at 0 , both must have the same supports, and the common support can contain no gaps. The derivation above, therefore, rules out all other possibilities.

\section{ACKNOWLEDGMENTS}

We are grateful to Roberto Burguet, Hsueh-Ling Huynh, Vijay Krishna, Cesar Martinelli, Preston McAfee, Ruqu Wang, and a referee for helpful suggestions; to Sumon Majumdar for research assistance; and to the Fullbright Program and the U.S. National Science Foundation for financial assistance. 


\section{REFERENCES}

Ashenfelter, O. (1989). "How Auctions Work for Wine and Art," J. Econ. Perspectives 3, 23-36.

Avery, C. (1994). "Strategic Jump Bidding in an English Auction," mimeo, Kennedy School of Government, Harvard University.

Bernhardt, D., and Scoones, D. (1994). "A Note on Sequential Auctions," Amer. Econ. Rev. 84, 653-657.

Bikhchandani, S. (1988). "Reputation in Repeated Second-Price Auctions," J. Econ. Theory 46, 97-119.

Gale, I., and Hausch, D. (1992). "Bottom Fishing and Declining Prices in Sequential Auctions," mimeo, Univ. of Wisconsin.

Lang, K., and Rosenthal, R. (1991). "The Contractor's Game," RAND J. Econ. 22, 329-338.

McAfee, P., and Vincent, D. (1993). "The Declining Price Anomaly," J. Econ. Theory 60, 191-212.

McMillan, J. (1994). "Selling Spectrum Rights," J. Econ. Perspectives 8, 145-162.

Milgrom, P. (1985). "The Economics of Competitive Bidding: A Selective Survey," in Social Goals and Social Organization (L. Hurwicz, D. Schmeidler and H. Sonnenschein, Eds.), pp. 216-220. Cambridge: Cambridge Univ. Press.

Milgrom, P., and Weber, R. (1982a). "A Theory of Auctions and Competitive Bidding," Econometrica 50, 1089-1122.

Milgrom, P., and Weber, R. (1982b). "A Theory of Auctions and Competitive Bidding, II," unpublished manuscript, Northwestern Univ.

Perry, M., and Reny, P. (1997). "On the Failure of the Linkage Principle in Multi-Unit Auctions," mimeo, Univ. of Pittsburgh.

Rosenthal, R. (1980). "A Model in which an Increase in the Number of Sellers Leads to a Higher Price," Econometrica 48, 1575-1579.

Rosenthal, R., and Wang, R. (1996). "Simultaneous Auctions with Synergies and Common Values," Games Econ. Behav. 17, 32-55.

Simon, L., and Zame, W. (1990). "Discontinuous Games and Endogenous Sharing Rules," Econometrica 58, 861-872.

Weber, R. (1983). "Multiple Object Auctions", in Auctions, Bidding and Contracting: Uses and Theory (R. Engelbrecht-Wiggans, M. Shubik, and R. M. Stark, Eds.), pp. 165-191. New York: New York Univ. Press. 